\title{
EXPERIMENTS WITH THE TWO-BEAM FUNNELING RFQ*
}

\author{
H. Zimmermann, A. Bechtold, A. Firjahn-Andersch, H. Liebermann, A. Schempp, \\ J. Thibus, E. Winschuh, \\ Institut für Angewandte Physik, Johann Wolfgang Goethe-Universität, \\ Robert-Mayer-Straße 2-4, D-60054 Frankfurt am Main, Germany
}

\begin{abstract}
High intensity accelerator concepts for Heavy Ion Inertial Fusion (HIIF) injectors require small emittance, high current and high energy beams. The improvement of brightness in such a driver linac is done by several funneling stages at low energies, in which two identically bunched ion beams are combined into a single beam with twice the frequency, current and brightness. For the Heavy-Ion Driven Ignition Facility (HIDIF) we have proposed the use of a two-beam accelerator structure which provides two beams within one cavity and a single r.f. deflector structure which bends the two beams to one common axis. The progress of the experiment and first beam-test results will be presented.
\end{abstract}

\section{INTRODUCTION}

The beam currents of linacs are limited by space charge effects and the focusing and transport capability of the accelerator.

Funneling is doubling the beam current by the combination of two bunched beams preaccelerated at a frequency $\mathrm{f}_{0}$ with an r.f. deflector to a common axis and injecting into another r.f. accelerator at frequency $2 * \mathrm{f}_{0}$.

By the use of the two-beam RFQ the two beams are brought very close together while they are still radially and longitudinally focused. Additional discrete elements like quadrupole-doublets and -triplets, debunchers and bending magnets, as they have been proposed in first funneling studies, are not necessary $[1,2,3]$. A short r.f. funneling deflector will be placed around the beam crossing position behind the RFQ. The layout of the proposed HIDIFinjector with two-beam RFQs in front of the first and second funneling sections is shown in figure 1 [4]. The HIDIF linac starts with 16 times 3 ion sources for three different ion species to allow so-called „telescoping“ at the final focus [5]. With four funneling stages the frequency has been increased from $12.5 \mathrm{MHz}$ to $200 \mathrm{MHz}$ accordingly [6].

For studies of the new two-beam RFQ structure and the r.f. deflector, the first two-beam funneling experiments will be carried out with $\mathrm{He}^{+}$-ions at low energies to facilitate ion source operation and beam diagnostics.

*Work supported by the BMBF

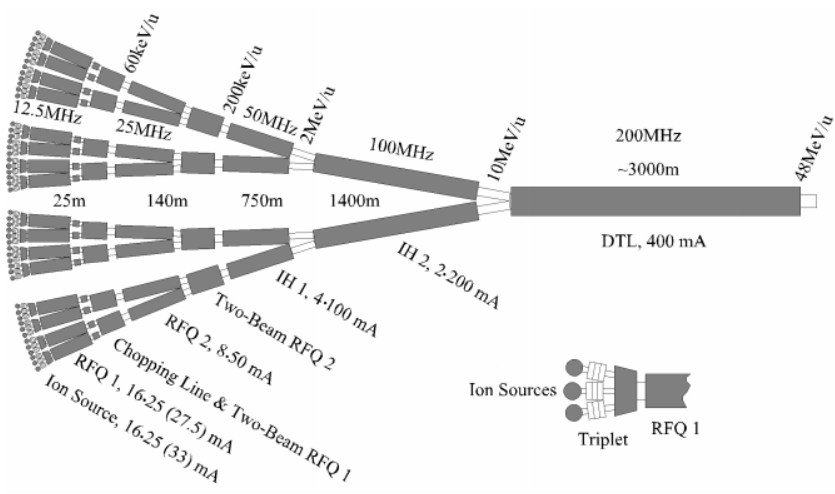

Figure 1: Layout of the $12.5 \ldots 200 \mathrm{MHz}$ linac system for $400 \mathrm{~mA}$ of $\mathrm{Bi}^{+}$.

Two small multicusp ion sources and electrostatic lenses, built by LBNL (Lawrence Berkeley National Laboratory) [7,8], are used. The ion sources and injection systems are attached directly on the front of the RFQ with an angle of $76 \mathrm{mrad}$, the angle of the beam axes of the two-beam RFQ.

Figure 2 shows a scheme of the experimental set-up of the two-beam funneling experiment.

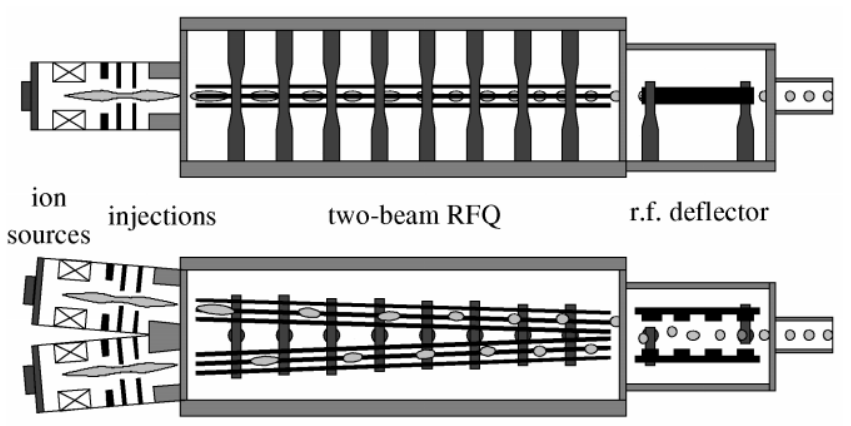

Figure 2: Experimental set-up of the two-beam funneling experiment.

\begin{tabular}{|r|c|c|}
\hline \multicolumn{1}{|l|}{} & $\mathbf{H e}^{+}$ & $\mathbf{B i}^{+}$ \\
\hline $\mathrm{f}_{0}[\mathrm{MHz}]$ & 54 & 12,5 \\
\hline Voltage $[\mathrm{kV}]$ & 10.5 & 180 \\
\hline $\mathrm{R}_{\mathrm{p}}$-value $[\mathrm{kOhm} \cdot \mathrm{m}]$ & 80 & 250 \\
\hline $\mathrm{Q}_{0}$-Value & 1800 & 5000 \\
\hline $\mathrm{T}_{\text {in }}[\mathrm{keV}]$ & 4 & 209 \\
\hline $\mathrm{T}_{\text {out }}[\mathrm{MeV}]$ & 0.16 & 12.54 \\
\hline Angle between beam axes $[\mathrm{mrad}]$ & 76 & 76 \\
\hline
\end{tabular}

Table 1: Main parameter of the experiment with the $\mathrm{He}^{+}$and the design parameters of a first HIDIF funneling stage for $\mathrm{Bi}^{+}$. 


\section{ION SOURCES AND INJECTION SYSTEMS}

To investigate the synchronous operation, the beam of both ion sources and injection systems have been tested on an emittance measurement device by different parameters of the ion sources and injection system. The measured normalized 90\% RMS-emittance is shown in fig. 3.

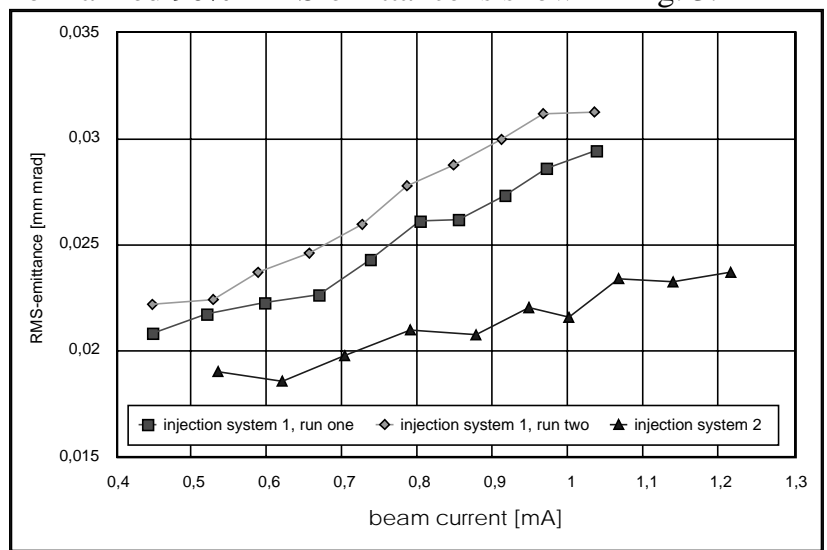

Figure 3: Normalized 90\% RMS-emittance of the two injection systems at different beam currents.

\section{THE TWO-BEAM RFQ}

The two-beam RFQ consists of two sets of quadrupole electrodes, where the beams are bunched and accelerated with a phase shift of $180^{\circ}$ between each bunch, driven by one resonant structure. With the use of identical RFQ electrode designs for both beam lines, the electrodes of one beam line are installed with a longitudinal shift of $2.55 \mathrm{~cm}$ (i.e. $\beta \lambda / 2$ at final energy) to achieve the $180^{\circ}$ phase shift between the beam bunches of each beam line. Figure 4 shows the with a fast faraday cup measured micro-bunch current of the two RFQs, which demonstrates the $180^{\circ}$ phase shift between the beam bunches.

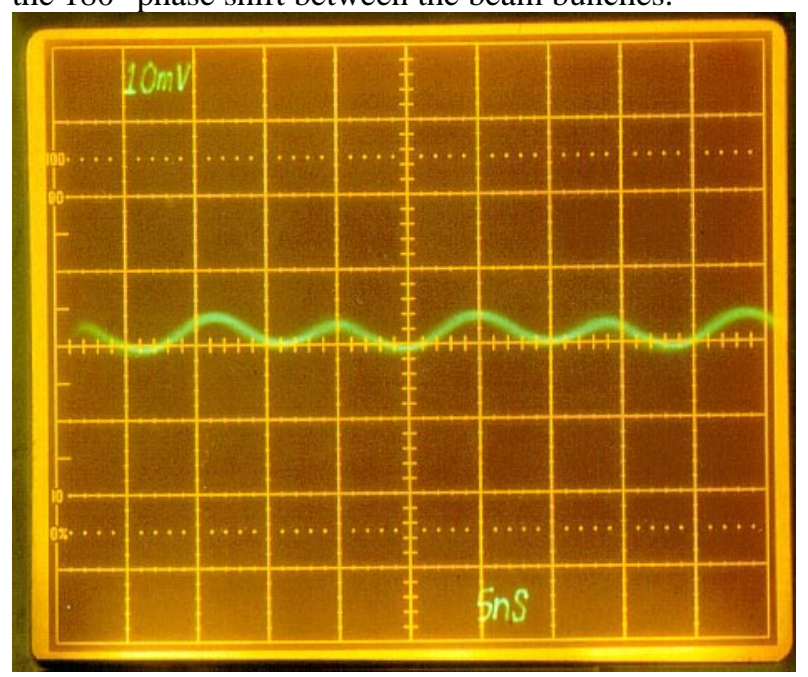

Figure 4: The measured micro-bunch current of the two RFQs demonstrate the $180^{\circ}$ phase shift between the beam bunches. The scale is $5 \mathrm{~ns} / \mathrm{div}$. horizontally.
In Figure 5 the measured energy spectra behind the RFQ are shown. The final beam-energy of $166 \mathrm{keV}$ is reached at an electrode voltage of $10 \mathrm{kV}$, which is the design voltage. Below $4 \mathrm{kV}$ electrode voltage the $\mathrm{He}^{+}$ beam is only transported and not accelerated. The energy is now the energy of the injection system of $4.15 \mathrm{keV}$.

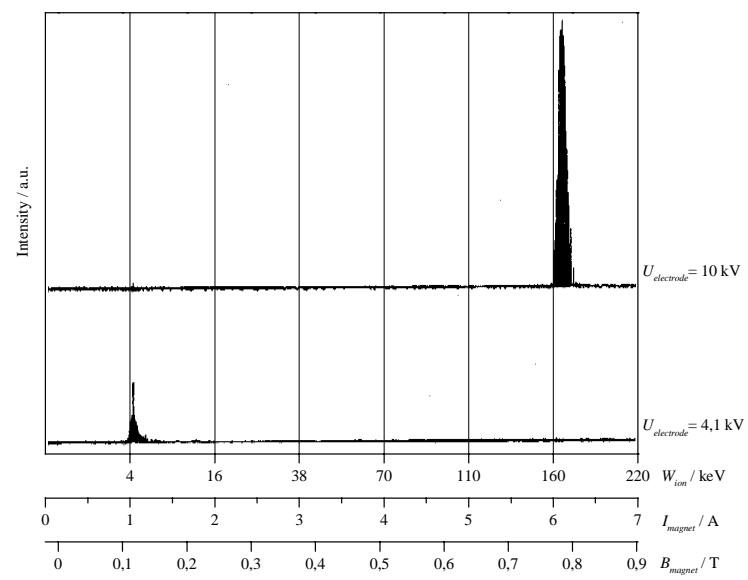

Figure 5: Energy spectra behind the RFQs. With the design voltage of $10 \mathrm{kV}$ the final beam-energy of $166 \mathrm{keV}$ is reached.

At the beam crossing position behind the two-beam RFQ emittance measurements have been done. Figure 6 shows the two measured RMS-emittances. The normalized $90 \%$ RMS-emittance of the two beams are 0.107 and $0.114 \mathrm{~mm} \mathrm{mrad}$, the deviation is about $6 \%$.

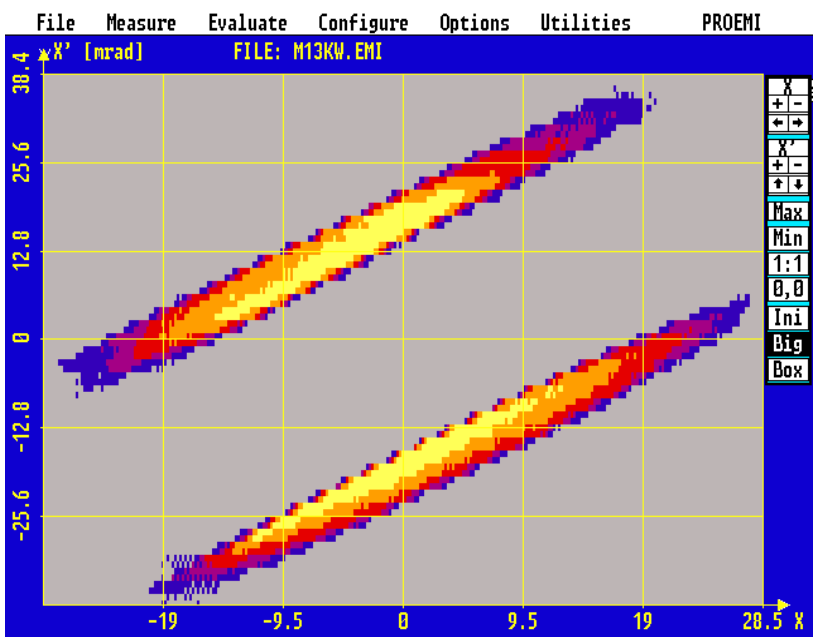

Figure 6: Measured emittances $1 \mathrm{~m}$ behind the RFQ at the place of beam crossing. The ellipses correspond to the $90 \%$ normalized RMS-emittances which are $\varepsilon_{\mathrm{RMS}}=0.107$ and $0.114 \mathrm{~mm}$ mrad for each beam.

\section{FUNNELING-DEFLECTOR}

For bending the two beams to a common axis, a funnelingdeflector is mounted directly behind RFQ. This device is like a plate capacitor with an electric field only in the 
bending plane. The crosspoint of the two beams is situated right in the middle of the deflector. As the bunches leave the RFQ with a phase shift of $180^{\circ}$ and with alternating angle ( $\pm 37.5 \mathrm{mrad})$, they are step by step deflected to angle zero, when the field is smaller in the case of wrong polarity. Figure 7 shows a simulation of the change in angle of the stable particle during the motion through the funneling-deflector. As the calculation shows, the optimum voltage for operation is about $3 \mathrm{kV}$. Figure 8 shows the scheme of the funneling-deflector.

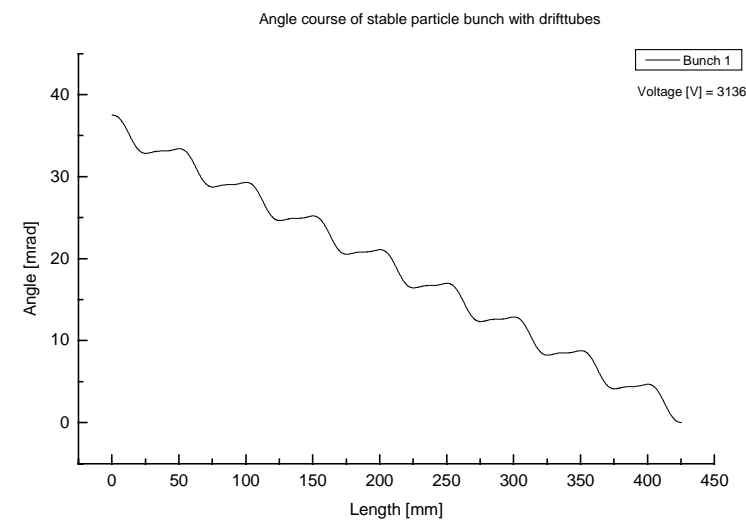

Figure 7: Motion of the stable-particle during bending in the funnelingdeflector.

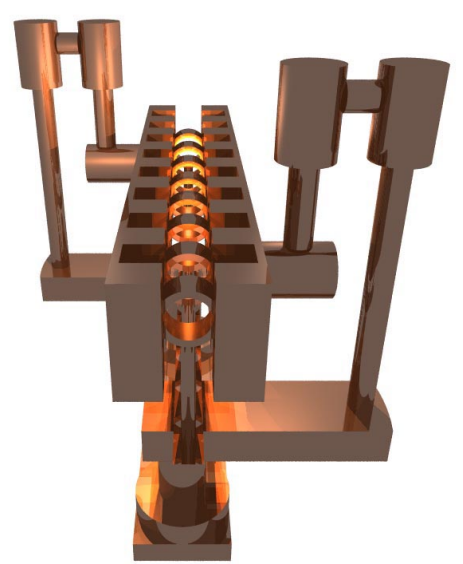

Figure 8: Picture of the funneling deflector. To reduce the E-field a drift tube is mounted between the plates each second cell.

To minimize the field in case of wrong polarity we decided to use drift tubes each second cell. The amplitude of the simulated E-field on the symmetrical axis of the deflector over the length of two cells is shown in Fig. 9.

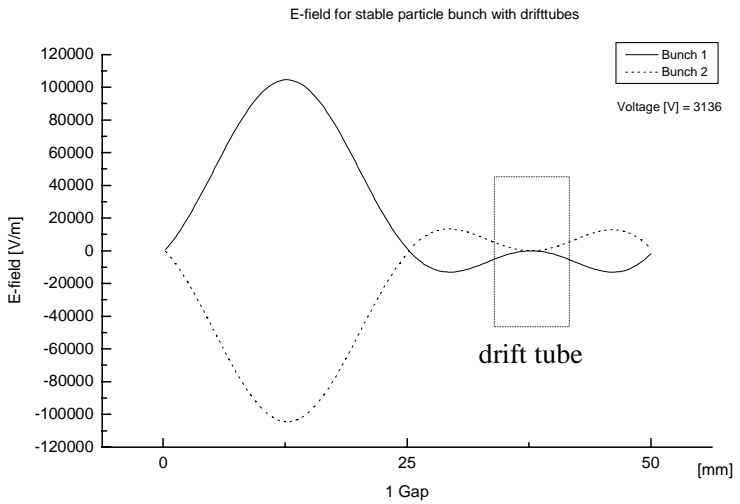

Figure 9: E-field in the funneling-deflector over the length of two cells.

\section{CONCLUSIONS}

The beam test results of the two-beam RFQ and the theoretical investigations on the funneling section have shown that the chosen set-up should be able to bend the two beams to a common axis. Next step will be the experimental test of the funneling-deflector.

\section{REFERENCES}

[1] K. Bongardt and D. Sanitz, Funneling of Heavy Ion Beams, Primary Report, Kernforschungszentrum Karlsruhe, 1104 02P14C (September 1982)

[2] J.F. Stovall, F.W. Guy, R.H. Stokes and T.P. Wangler, Beam Funneling Studies at Los Alamos, Nucl. Instr, and Meth. A278 (1989) p143

[3] K.F. Johnson, O.R. Sander, G.O. Bolmer, J.D. Gilpatrick, F.W Guy, J.H. Marquardt, K. Saadatmand, D. Dandoval and V. Yuan, A Beam Funnel Demonstration: Experiment and Simulation, Particle Accelerators, Vols. 37-38 (1992) p. 261

[4] A. Firjahn-Andersch, J. Madlung, A. Schempp, H. Zimmermann A two-beam RFQ for ion beam funneling, Proc. (International) Particle Accelerator Conference 1997, p. 1081-1083

[5] M. Basco, M. Churazov, D. Koshkarev, Fusion Engineering and Design 32-33 (1996) p. 73.

[6] A. Schempp, The injector for the HIDIF driver linac, Nuclear Instruments and Methods in Physics Research A415 (1998) 209-217

[7] K.N. Leung, Multicusp Ion Sources, Rev. Sci. Instrum. 65(4) (1994) p. 1165.

[8] R. Keller in: The Physics and Technology of Ion Sources, Edited by I. G. Brown, Wiley-Interscience Publication, New York.

[9] A. Schempp, Design of Compact RFQs, Proc. Linear Accelerator Conference 1996, CERN 96-07, p. 53. 